\title{
Prevalence of Osteosarcopenia and Its Association with Cardiovascular Risk Factors in Iranian Older People: Bushehr Elderly Health (BEH) Program
}

\author{
Noushin Fahimfar ${ }^{1} \cdot$ Farbod Zahedi Tajrishi $^{2} \cdot$ Safoora Gharibzadeh ${ }^{3} \cdot$ Gita Shafiee $^{4} \cdot$ Kiarash Tanha $^{5}$. \\ Ramin Heshmat ${ }^{4} \cdot$ Iraj Nabipour $^{6} \cdot$ Alireza Raeisi $^{7} \cdot$ Ali Jalili $^{1} \cdot$ Bagher Larijani $^{8} \cdot$ Afshin Ostovar $^{1} \mathbb{C}$
}

Received: 23 June 2019 / Accepted: 6 December 2019 / Published online: 17 December 2019

(c) Springer Science+Business Media, LLC, part of Springer Nature 2019

\begin{abstract}
Osteosarcopenia is an increasingly recognized geriatric syndrome with a considerable prevalence which increases morbidity and mortality. Although osteosarcopenia is a result of age-related deterioration in muscle and bone, there are many risk factors that provoking osteosarcopenia. These risk factors should be considered by the clinicians to treat osteosarcopenia. We assessed the link between osteosarcopenia and conventional risk factors of cardiovascular diseases. This study was a crosssectional study that has been conducted within the framework of Bushehr Elderly Health (BEH) program stage II in which participants aged $\geq 60$ years were included. Osteopenia/osteoporosis was defined as a $t$-score $\leq-1.0$ standard deviation below the mean values of a young healthy adult. We defined sarcopenia as reduced skeletal muscle mass plus low muscle strength and/or low physical performance. Osteosarcopenia was considered as the presence of both osteopenia/osteoporosis and sarcopenia. We estimated the age-standardized prevalence of osteosarcopenia for men and women, separately. Using modified Poisson regression analysis, adjusted prevalence ratio (PR) with 95\% CI was used to show the measure of associations in the final model. Among 2353 participants, 1205 (51.2\%) were women. Age-standardized prevalence of osteosarcopenia was 33.8 (95\% CI 31.0-36.5) in men and 33.9 (30.9-36.8) in women. In both sexes, the inverse association was detected with body mass index and having osteosarcopenia (PR 0.84, 95\% CI 0.81-0.88 in men and 0.77, 95\% CI 0.74-0.80 in women). In both sexes, high-fat mass was positively associated with osteosarcopenia [PR 1.46 (95\% CI 1.11-1.92) in men, and 2.25 (95\% CI 1.71-2.95) in women]. Physical activity had a significant inverse association in men ( $P R=0.64,95 \%$ CI $0.46,0.88$ ), but not in women. Diabetes was also showed a direct association with osteosarcopenia in men (PR 1.33, 95\% CI 1.04-1.69). No associations were detected between the lipid profiles and osteosarcopenia. Results demonstrated a high prevalence of osteosarcopenia in both sexes suggesting a high disease burden in a rapidly aging country. Lifestyle and socioeconomic factors, as well as chronic diseases, were significantly associated with osteosarcopenia.
\end{abstract}

Keywords Prevalence $\cdot$ Osteosarcopenia $\cdot$ Osteoporosis $\cdot$ Sarcopenia $\cdot$ Elderly $\cdot$ Iran

Afshin Ostovar

aostovar@tums.ac.ir

1 Osteoporosis Research Center, Endocrinology and Metabolism Clinical Sciences Institute, Tehran University of Medical Sciences, No. 10-Jalal-e-ale-ahmad St, Chamran Hwy, P. O. Box, Tehran 14117-13137, Iran

2 School of Medicine, Babol University of Medical Sciences, Babol, Iran

3 Department of Epidemiology and Biostatistics, Pasteur Institute of Iran, Tehran, Iran

4 Chronic Diseases Research Center, Endocrinology and Metabolism Population Sciences Institute, Tehran University of Medical Sciences, Tehran, Iran
5 Department of Biostatistics, School of Public Health, University of Medical Sciences, Tehran, Iran

6 The Persian Gulf Marine Biotechnology Research Center, The Persian Gulf Biomedical Sciences Research Institute, Bushehr University of Medical Sciences, Bushehr, Iran

7 School of Medicine, Shiraz University of Medical Sciences, Shiraz, Iran

8 Endocrinology and Metabolism Research Center, Endocrinology and Metabolism Clinical Sciences Institute, Tehran University of Medical Sciences, Tehran, Iran 


\section{Introduction}

Osteosarcopenia is an increasingly recognized geriatric syndrome, defined by the presence of sarcopenia which is a gradual build-up of body fat and a resultant reduction in lean muscle mass and strength and also osteopenia/ osteoporosis. In an era with a globally growing elderly and frail population, the condition is receiving further attention as multiple large studies have linked both osteopenia and sarcopenia with morbidity and mortality in this age group [1-4].

There is plenty of evidence on the prevalence, risk factors, and outcomes of osteoporosis as well as a growing body of evidence on sarcopenia; however, only a limited number of publications are available on osteosarcopenia as a syndrome addressing the cross-talk between bone and muscle [5]. A few studies on the prevalence of osteosarcopenia in different countries have reported the prevalence rates of $12.5 \%$ to $40 \%$ among different elderly populations [6].

In addition to weakness and disability, osteosarcopenia is linked with cardiovascular diseases (CVDs) and their associated risk factors such as hyperlipidemia, obesity, type 2 diabetes mellitus, and hypertension [7-10]. There is, however, some contradictory evidence rejecting such a direct association between CVD and osteosarcopenia [10-15]. Although these studies had their own limitations [16], they have warranted the necessity of more investigations to produce further evidence and help end the controversy.

In the present study, we analyzed the true link between osteosarcopenia and CVD risk factors in a large population of elderly over 60 years old living in Bushehr, Iran. We used baseline data from the second stage of the Bushehr Elderly Health (BEH) program, which was initiated in October 2015 [17].

\section{Materials and Methods}

\section{Study Population}

This cross-sectional study was conducted within the framework of BEH program being conducted since 2013. The BEH program is a prospective, population-based cohort aiming at determining the prevalence and risk factors of non-communicable diseases among a representative sample of the elderly urban population residing in Bushehr, southern Iran [18]. In stage II of the study, 2426 participants aged $\geq 60$ years were included to estimate the prevalence of musculoskeletal disorders, cognitive impairment, and their risk factors, while also evaluating the associations between these entities and certain outcomes during future follow-ups. The rationale for and methodology was described in detail elsewhere [17]. The study was approved by the Research Ethics Committee of both Bushehr University of Medical Sciences and Endocrinology and Metabolism Research Institute. After an explanation of the study objectives and protocols, written informed consent was obtained from all participants.

\section{Data Collection}

Using a valid questionnaire, subjects were privately interviewed by trained interviewers to collect information on sociodemographic status, lifestyle-associated factors, general health, medical history, and medication use, as well as mental and functional health [17].

The height and weight of the participants were measured using a fixed stadiometer and a digital scale, with shoes removed and the participants wearing light clothing. Body mass index (BMI) was calculated as weight $(\mathrm{kg})$ divided by squared height $\left(\mathrm{m}^{2}\right)$. All measurements were read to the nearest $0.1 \mathrm{~cm}$. Following $15 \mathrm{~min}$ in the sitting position, two measurements of systolic and diastolic blood pressure were taken on the right arm, and the mean of the two measurements was considered as the participant's blood pressure.

Patients' lipid profile and blood glucose were measured by assessing venous samples, drawn after overnight fasting. Using the enzymatic colorimetric method with cholesterol esterase and cholesterol oxidase, total cholesterol (TC) was determined. Details for the measurements of fasting plasma glucose (FPG), high-density lipoprotein (HDL-C), low-density lipoprotein cholesterol (LDL-C), and triglyceride (TG) were reported elsewhere [17].

Physical activity level was assessed by a validated questionnaire in which nine different metabolic equivalents of task (MET) levels were ranged on a scale from sleep/rest to high-intensity physical activities [19, 20].

Body composition was measured using dual X-ray absorptiometry (DXA, Discovery WI, Hologic, Inc., USA). Using this device, bone mineral density, fat mass, and muscle mass were measured. The bone mineral density of the lumbar spine (L1-4) and total hip were measured in the correct position. Appendicular skeletal muscle mass (ASM) for each participant was derived as the sum of upper and lower limb muscle mass. The skeletal muscle mass index (SMI) was defined as ASM/height ${ }^{2}\left(\mathrm{~kg} / \mathrm{m}^{2}\right)$. Muscle strength was measured by handgrip strength, using a digital dynamometer. The measurement was carried out three times for each hand, and maximum grip strength was calculated by taking the average of the highest measurement from both hands. Usual walking speed $(\mathrm{m} / \mathrm{s})$ on a $4.57-\mathrm{m}$ course was used as an objective measure of physical performance. 


\section{Definition of Terms}

Osteopenia/ osteoporosis was defined using the WHO standard criteria of $t$-score less than -1.0 standard deviation (SD) in either the femoral neck, lumbar spine, or total hip [21]. Sarcopenia was defined as reduced skeletal muscle mass plus low muscle strength and/or low physical performance [22, 23]. In a recent study, reference data from a normative Iranian population are available for detecting sarcopenia. Based on these data, the cut-off values for low SMI were $7.0 \mathrm{~kg} / \mathrm{m}^{2}$ and $5.4 \mathrm{~kg} / \mathrm{m}^{2}$ among men and women, respectively [24].

The muscle strength was handgrip strength $<26 \mathrm{~kg}$ for men and $<18 \mathrm{~kg}$ for women; while the cut-off value for low physical performance was a usual walking speed $<0.8 \mathrm{~m} / \mathrm{s}$ for both genders [23]. Using these cut-off points, sarcopenic individuals were identified. Therefore, osteosarcopenia has been defined as the presence of sarcopenia and osteopenia/ osteoporosis.

Type 2 diabetes mellitus was ascertained among participants who had FPG $\geq 126 \mathrm{mg} / \mathrm{dl}$ or taking anti-diabetic medication. Hypertension was defined as SBP $\geq 140 \mathrm{mmHg}$ or DBP $\geq 90 \mathrm{mmHg}$ or taking any anti-hypertensive medications. Hypercholesterolemia was defined as $\mathrm{TC} \geq 200 \mathrm{mg} / \mathrm{dl}$, high LDL-C as $>110 \mathrm{mg} / \mathrm{dl}$, low HDL-C as $<40 \mathrm{mg} / \mathrm{dl}$ for men and $<50 \mathrm{mg} / \mathrm{dl}$ for women, and hypertriglyceridemia as $\mathrm{TG} \geq 150 \mathrm{mg} / \mathrm{dl}$ [25]. High-fat mass was outlined as total body percent fat $>30$ for men and $>40$ for women [26]. Current smokers were defined as participants who were smoking cigarettes daily or occasionally as well as those who used water pipes or pipes. In addition, participants were grouped as low physical activity or active, according to the MET values $[20,27]$.

\section{Statistical Analysis}

The data were expressed as mean $( \pm \mathrm{SD})$ for continuous and the percentage for categorical variables. The osteosarcopenic and non-osteosarcopenic populations were compared for the baseline characteristics using Pearson's $\chi^{2}$ test for the categorical and independent sample $t$-test for continuous variables. All comparisons were conducted in men and women, separately. The prevalence of osteosarcopenia in different age groups was estimated in both sexes. The crude prevalence of osteosarcopenia in the whole population was estimated by sex. Direct standardization, using the population distribution of Iranian census data of 2016, was used to measure the age-standardized prevalence rates in men and women. We assessed the association of potential cardiovascular variables on osteosarcopenia by a modified Poisson regression model.

Age, education, smoking, physical activity, BMI, hypertension, diabetes, hypercholesterolemia, hypertriglyceridemia, low HDL-C, high LDL-C, and high-fat mass were considered as the main set of variables. To select the best model from all possible subsets and avoid overfitting, best subset method with the Akaike Information Criterion (AIC) was used. The multi-collinearity between variables was checked and the adjusted prevalence ratio (Adjusted PR) with $95 \% \mathrm{CI}$ was used as the measure of associations in the final model. We considered the $p$-values $<0.05$ as statistically significant. Stata statistical package, release 14 , was used to perform the statistical analyses.

\section{Results}

After excluding the participants with missing values for osteosarcopenia $(N=48)$, and having any kind of cancer $(N=25)$, a total of 2353 participants (1205 women) were included in the present study. In all, 1988 (84.5\%) participants had osteopenia/osteoporosis and sarcopenia was detected in 779 (33.3\%) of the study population. Osteosarcopenia was diagnosed in 369 (30.6\%) of women and 339 (29.5\%) of men. Table 1 demonstrates the baseline characteristics of the participants by the status of osteosarcopenia in men and women. Age, BMI, physical activity, hypertriglyceridemia, high-fat mass, and education were significantly different in participants with and without osteosarcopenia in both sexes, and also the prevalence of hypertension and smoking status in women (all $p$-values $<0.05$ ).

Figure 1 shows the prevalence of osteosarcopenia among four different age groups in men and women. As it highlights, in age below 75 years, the prevalence of osteosarcopenia is higher in women, although the differences are not significant in all groups ( $p$-values were $0.055,0.178$, and 0.049 in 60-64 years, 65-69, and 70-74 years, respectively; however, among the oldest population ( $\geq 75$ years), the prevalence was higher in men $(59 \%$ vs. $48 \%, p$-value 0.015). In both sexes, the prevalence of osteosarcopenia increased by age, while the slope was higher in men (more than fourfold increase from $14.3 \%$ in aged $60-64$ years to $59.4 \%$ in aged $\geq 75$ years in men and more than twofold increase from $20.3 \%$ in aged $60-64$ years to $48.3 \%$ in aged $\geq 75$ years in women; $p$-interaction 0.019$)$. The agestandardized PR of osteosarcopenia in both sexes was nearly the same $33.8 \%$ (95\% CI 31.0-36.5\%) in men and $33.9 \%$ (95\% CI 30.9-36.8\%) in women.

Table 2 presents the results of the modified Poisson regression model to define the associate variables with osteosarcopenia. The best subset model includes BMI, high-fat mass, diabetes and education in both sexes, additional to the age and physical activity that were only significant in men. BMI was inversely associated with osteosarcopenia, as having osteosacropenia was less likely with increasing BMI (PR 0.84, 95\% CI 0.81-0.88 in men and PR $0.77,95 \%$ CI $0.74-0.80$ in women). Moreover, 
Table 1 Demographic characteristics of the participants by sex; Bushehr Elderly Health (BEH) program

\begin{tabular}{|c|c|c|c|}
\hline Continuous variables & $\begin{array}{l}\text { Osteosarcopenia }^{+} \\
\text {Mean }(95 \% \text { CI })\end{array}$ & $\begin{array}{l}\text { Osteosarcopenia } \\
\text { Mean }(95 \% \mathrm{CI})\end{array}$ & $p$-value \\
\hline \multicolumn{4}{|l|}{ Women } \\
\hline Age & $71.2(70.5-71.9)$ & $68.3(67.9-68.7)$ & $<0.001$ \\
\hline Body mass index & $24.2(23.9-24.6)$ & $30.5(30.2-30.8)$ & $<0.001$ \\
\hline Years of schooling ${ }^{\mathrm{a}}$ & $0(0-4.5)$ & $2(0-6)$ & $<0.001$ \\
\hline Categorical variables & $N(\%)$ & $N(\%)$ & \\
\hline \multicolumn{4}{|l|}{ Women } \\
\hline Diabetes mellitus & $123(33.3)$ & $317(38.0)$ & 0.12 \\
\hline Hypertension & $257(69.7)$ & $649(77.6)$ & 0.003 \\
\hline Current smoking & $76(21.2)$ & $124(15.1)$ & 0.010 \\
\hline Physical activity & 69 (18.7) & $208(24.9)$ & 0.019 \\
\hline Low HDL & $196(53.1)$ & $490(58.6)$ & 0.07 \\
\hline Hypertriglyceridemia & $95(25.8)$ & $318(38.0)$ & $<0.001$ \\
\hline Hypercholesterolemia & $152(41.2)$ & $321(38.4)$ & 0.36 \\
\hline High-fat mass & $234(63.4)$ & $705(84.6)$ & $<0.001$ \\
\hline Continuous variables & Mean (SD) & Mean (SD) & \\
\hline \multicolumn{4}{|l|}{ Men } \\
\hline Age & $73.3(72.5-74.1)$ & $67.9(67.5-68.3)$ & $<0.001$ \\
\hline Body mass index & $23.9(23.6-24.3)$ & $27.1(26.9-27.4)$ & $<0.001$ \\
\hline Years of schooling $^{\mathrm{a}}$ & $6(0-10)$ & $8(4-12)$ & $<0.001$ \\
\hline Categorical variables & $N(\%)$ & $N(\%)$ & \\
\hline \multicolumn{4}{|l|}{ Men } \\
\hline Diabetes mellitus & $99(29.3)$ & $242(30.0)$ & 0.82 \\
\hline Hypertension & $236(69.6)$ & $573(70.09)$ & 0.66 \\
\hline Current smoking & $80(24.1)$ & $151(19.4)$ & 0.07 \\
\hline Physical activity & $46(13.6)$ & $223(27.6)$ & $<0.001$ \\
\hline Low HDL & $125(36.9)$ & $331(40.9)$ & 0.20 \\
\hline Hypertriglyceridemia & $76(22.4)$ & $252(31.2)$ & 0.003 \\
\hline Hypercholesterolemia & $82(24.2)$ & $207(25.6)$ & 0.61 \\
\hline High-fat mass & $181(53.4)$ & $518(64.8)$ & $<0.001$ \\
\hline
\end{tabular}

$p$-values in bold indicate the significant results

Continuous variables shown as mean \pm SD. Independent $t$-test and Pearson's $\chi^{2}$ test were applied for quantitative and qualitative variables respectively

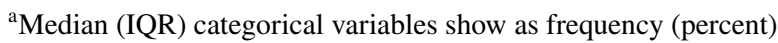

each year of schooling was associated with a 3\% lower prevalence of osteosarcopenia in men (PR 0.97, 95\% CI 0.95-0.99). Although the year of schooling showed the same association with osteosarcopenia in women, it was not statistically significant. Osteosarcopenia was less likely in men with physical activity $(P R=0.64,95 \%$ CI 0.46 , 0.88 ) but no significant effect was detected in women. In both sexes, osteosarcopenia was more likely in individuals with high-fat mass ( $\mathrm{PR}=1.46,95 \% \mathrm{CI} 1.11-1.92)$ in men and $(\mathrm{PR}=2.25,95 \% \mathrm{CI} 1.71-2.95)$ in women. Having osteosarcopenia was more likely in diabetic men (PR 1.33, 95\% CI 1.04-1.69), but not in women.

\section{Discussion}

Our study found a high prevalence of osteosarcopenia among a large sample of the elderly population. Though the difference between genders was not statistically significant in all groups, the condition was more prevalent in women of all age categories, except among the oldest group ( $\geq 75$ years), where it was more prevalent among men. Our findings primarily suggest that independent from gender, BMI has an inverse association with osteosarcopenia, whereas high-fat mass was directly linked to a nearly 


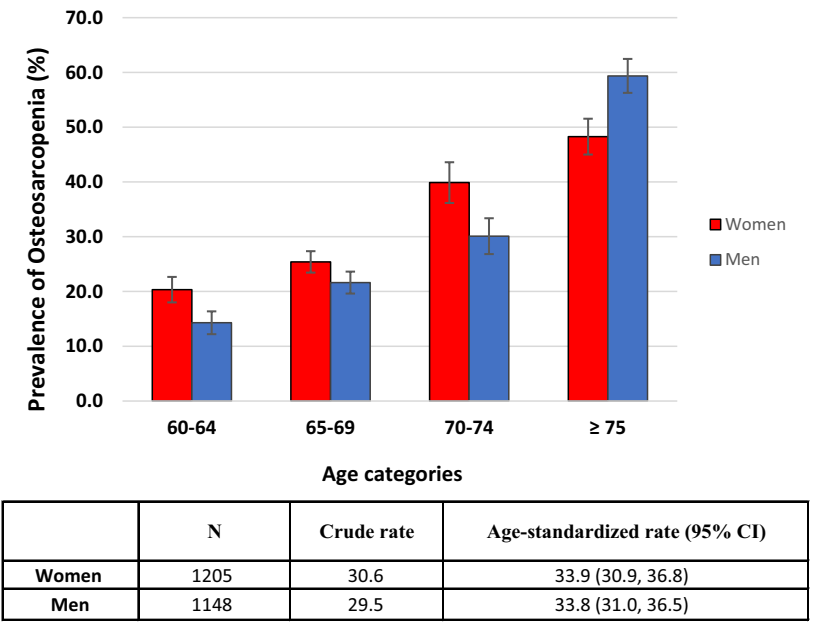

Fig. 1 Crude and age-standardized prevalence rates of osteosarcopenia in men and women; Bushehr Elderly Health (BEH) program

Table 2 Correlates of having osteosarcopenia in men and women; Bushehr Elderly Health (BEH) program

\begin{tabular}{lcc}
\hline & Adjusted PR $(95 \%$ CI $)$ & $p$-value \\
\hline Women & - & \\
Age & $0.97(0.95-1.00)$ & - \\
Education & - & 0.07 \\
Physical activity & $1.22(0.97-1.52)$ & - \\
Diabetes & $\mathbf{0 . 7 7}(\mathbf{0 . 7 4 - 0 . 8 0 )}$ & 0.08 \\
Body mass index & $\mathbf{2 . 2 5}(\mathbf{1 . 7 1 - 2 . 9 5})$ & $<\mathbf{0 . 0 0 1}$ \\
High-fat mass & & $<\mathbf{0 . 0 0 1}$ \\
Men & $\mathbf{1 . 0 5}(\mathbf{1 . 0 4}-\mathbf{1 . 0 7})$ & \\
Age & $\mathbf{0 . 9 7}(\mathbf{0 . 9 5 - 0 . 9 9 )}$ & $<\mathbf{0 . 0 0 1}$ \\
Education & $\mathbf{0 . 6 4}(\mathbf{0 . 4 6 - 0 . 8 8})$ & $\mathbf{0 . 0 2}$ \\
Physical activity & $\mathbf{1 . 3 3}(\mathbf{1 . 0 4}-\mathbf{1 . 6 9})$ & $\mathbf{0 . 0 0 6}$ \\
Diabetes & $\mathbf{0 . 8 4}(\mathbf{0 . 8 1}-\mathbf{0 . 8 7})$ & $\mathbf{0 . 0 2 2}$ \\
Body mass index & $\mathbf{1 . 4 6}(\mathbf{1 . 1 1}-\mathbf{1 . 9 2})$ & $\mathbf{0 . 0 0 1}$ \\
High-fat mass & & $\mathbf{0 . 0 0 7}$ \\
\hline
\end{tabular}

$p$-values in bold indicate the significant results

$P R$ prevalence ratio, $C I$ confidence interval

twofold increased prevalence of the condition, making it the strongest associated factor we evaluated.

The results of the present study were mainly in line with previous studies that had generally suggested endocrine metabolism dysfunctions such as obesity and diabetes, and also physical inactivity as factors influencing both muscle and bone status [28]. Another study on postmenopausal women showed the association of low skeletal muscle mass with metabolic syndrome, independent of other metabolic risk factors; as in the sarcopenia group, the odds of having metabolic syndrome were nearly twice, compared to the normal group [29].
We found nearly $34 \%$ of our study population to be osteosarcopenic. Previous studies on the prevalence of osteosarcopenia as an independent condition among the elderly are few and consist of heterogeneous populations. A study on hip fracture patients in Korea reported a prevalence of $27.2 \%$ for osteosarcopenia [30], while studies on the German prefrail [31] and Australian [32] and Chinese [33] communitydwelling elderly yielded prevalence rates of $28 \%, 40 \%$, and $13 \%$, respectively.

Our study revealed that BMI and high-fat mass are independently associated with the likelihood of osteosarcopenia in both men and women. Some studies emphasized the importance of the independent role of BMI and waist circumference (WC) in clinical practice [34]. In our study, compared with WC, fat mass was a stronger associated variable that remained in the model, independent of BMI. The protective role of greater BMI levels in association with the harmful effect of high-fat mass highlights the importance of lean muscle mass. The function of bone, muscle, and adipose tissues are closely linked to one another via mechanical and neuro-hormonal pathways. Any disturbance in these communication mechanisms could result in a simultaneous dysfunction in these tissues [35]. Moreover, studies have demonstrated that atrophied muscle and bone are replaced by adipose tissue in elderly osteosarcopenic patients [35, 36]. These findings possibly explain why a higher level of BMI, with more lean muscle mass and less fat mass, reduces the risk of osteosarcopenia.

A higher proportion of muscle to fat mass has also been previously associated with a lower risk of cardio-metabolic and cardiovascular complications. For instance, in a systematic review of the literature, Gusmão-Sena et al. demonstrated that "sarcopenic obesity" is linked with cardio-metabolic and CVDs as well as their risk factor [37]. Although their study was limited by the heterogeneous definitions for sarcopenic obesity that exist in the literature, they well revealed that coexisting of sarcopenia and high-fat mass is a risk factor worth further studying. Atkins et al. studied a cohort consisting of 4252 men aged 60-79 years and similarly found that sarcopenic obesity was associated with higher all-cause mortality [38]. In our study, we found diabetes to be associated with osteosarcopenia in men. Although the association between diabetes and osteosarcopenia was not statistically significant, the magnitude of the PR was considerable (PR 1.22).

Though previous studies have revealed that type $1 \mathrm{DM}$ can result in osteopenia/osteoporosis in adulthood, the link between type $2 \mathrm{DM}$ and osteoporosis is not well recognized [39]. Although higher trabecular bone density has been reported in diabetic patients, cortical bone density is lower which results in low bone quality [40] and increased risk of fracture among diabetic patients. Regarding sarcopenia, insulin therapy in diabetes could cause weight gain [41]. 
Furthermore, insulin resistance in these patients could result in accelerated muscle loss primarily because of metabolic and hormonal abnormalities [42]. These changes may make diabetic patients susceptible to osteosarcopenic obesity. However, current evidence cannot explain the different prevalence of osteosarcopenia between diabetic men and women. Since the evidence on the connection between diabetes and osteosarcopenia is lacking, further studies are needed.

We couldn't find any association between osteosarcopenia and smoking and also lipid profiles.

Education was another factor to yield different results between men and women. We found that osteosarcopenia was less prevalent among educated elderly men. However, it did not statistically demonstrate a protective role against the condition in women. Theoretically, higher education is associated with a better lifestyle, including paying more attention to physical activity and a healthier diet compared with the less educated $[43,44]$. Some unobserved determinants of health behaviors may also be related to the education. Almost half of the elderly women in our study population had no education, resulting in less heterogeneity in this regard, which may explain the lack of association among women observed in this study.

\section{Strengths and Limitations}

This study with a large sample size from a population-based study supplied information on osteosarcopenia. Since osteosarcopenia is a newly-introduced condition, we did not come across many studies on osteosarcopenia in our search; instead, we found a handful of studies assessing the association between cardio-metabolic risk factors and either osteopenia/osteoporosis or sarcopenia.

Our study showed a positive association between fat mass and osteosarcopenia; however like many other studies using DXA scan, we were unable to differentiate and quantify marrow adipose tissue, intermuscular adipose tissue, and intramuscular fat or intramyocellular lipid since DXA only quantifies lean mass [45]. Further studies using peripheral quantitative $\mathrm{CT}$ (pQCT), high resolution $\mathrm{pQCT}$, magnetic resonance imaging (MRI), or peripheral MRI are needed to quantify ectopic fat and investigate its association with osteosarcopenia, independent of total body fat mass.

Since the osteosarcopenia consists of two different diseases, the associations detected can be influenced by the both direction and power of the association of risk factor with each of disease components. Therefore, more studies should be performed to better understand the epidemiology and nature of this syndrome. Furthermore, as our study was a cross-sectional one, causal inferences cannot be made and further prospective studies are needed to clarify the underlying causes.

\section{Conclusions}

Osteosarcopenia is a prevalent condition among a large proportion of Iranian elderly population. Independent from gender, BMI is inversely associated with osteosarcopenia, while high-fat mass is directly associated with the condition. Diabetes increased the prevalence of osteosarcopenia in men.

\section{Compliance with Ethical Standards}

Conflict of interest Noushin Fahimfar, Farbod Zahedi Tajrishi, Safoora Gharibzadeh, Gita Shafiee, Kiarash Tanha, Ramin Heshmat, Iraj Nabipour, Alireza Raeisi, Ali Jalili, Bagher Larijani, and Afshin Ostovar declare that they have no conflict of interest.

Human and Animal Rights and Informed Consent The BEH program was received the ethical approval of both Bushehr University of Medical Sciences and Endocrinology and Metabolism Research Institute. Informed consent was obtained from all participants included in the BEH program.

\section{References}

1. Narici MV, Maffulli N (2010) Sarcopenia: characteristics, mechanisms and functional significance. Br Med Bull 95:139-159

2. Consensus development conference: prophylaxis and treatment of osteoporosis (1991). Am J Med 90(1):107-110

3. World Health Organization (2019) Interesting facts about ageing. https://www.who.int/ageing/about/facts/en. Accessed 25 Jan 2019

4. Hassan EB, Duque G (2017) Osteosarcopenia: a new geriatric syndrome. Aust Fam Physician 46(11):849-853

5. Reginster JY, Beaudart C, Buckinx F, Bruyere O (2016) Osteoporosis and sarcopenia: two diseases or one? Curr Opin Clin Nutr Metab Care 19(1):31-36

6. Yoo JI, Ha YC (2018) Review of epidemiology, diagnosis, and treatment of osteosarcopenia in Korea. J Bone Metab 25(1):1-7

7. Varenna M, Manara M, Galli L, Binelli L, Zucchi F, Sinigaglia L (2013) The association between osteoporosis and hypertension: the role of a low dairy intake. Calcif Tissue Int 93(1):86-92

8. Dominguez LJ, Muratore M, Quarta E, Zagone G, Barbagallo M (2004) Osteoporosis and diabetes. Reumatismo 56(4):235-241

9. Migliaccio S, Greco EA, Fornari R, Donini LM, Lenzi A (2011) Is obesity in women protective against osteoporosis? Diabetes Metab Syndr Obes 4:273-282

10. Aubertin-Leheudre M, Lord C, Goulet ED, Khalil A, Dionne IJ (2006) Effect of sarcopenia on cardiovascular disease risk factors in obese postmenopausal women. Obesity (Silver Spring) 14(12):2277-2283

11. Sanada K, Miyachi M, Tanimoto M, Yamamoto K, Murakami H, Okumura $\mathrm{S}$ et al (2010) A cross-sectional study of sarcopenia in Japanese men and women: reference values and association with cardiovascular risk factors. Eur J Appl Physiol 110(1):57-65

12. Davison KK, Ford ES, Cogswell ME, Dietz WH (2002) Percentage of body fat and body mass index are associated with mobility limitations in people aged 70 and older from NHANES III. J Am Geriatr Soc 50(11):1802-1809

13. Goulet ED, Lord C, Chaput JP, Aubertin-Leheudre M, Brochu M, Dionne IJ (2007) No difference in insulin sensitivity between 
healthy postmenopausal women with or without sarcopenia: a pilot study. Appl Physiol Nutr Metab 32(3):426-433

14. Oterdoom LH, Gansevoort RT, Schouten JP, de Jong PE, Gans RO, Bakker SJ (2009) Urinary creatinine excretion, an indirect measure of muscle mass, is an independent predictor of cardiovascular disease and mortality in the general population. Atherosclerosis 207(2):534-540

15. Metter EJ, Talbot LA, Schrager M, Conwit R (2002) Skeletal muscle strength as a predictor of all-cause mortality in healthy men. J Gerontol A 57(10):B359-B365

16. Chin SO, Rhee SY, Chon S, Hwang YC, Jeong IK, Oh S et al (2013) Sarcopenia is independently associated with cardiovascular disease in older Korean adults: the Korea National Health and Nutrition Examination Survey (KNHANES) from 2009. PLoS ONE 8(3):e60119

17. Shafiee G, Ostovar A, Heshmat R, Darabi H, Sharifi F, Raeisi A et al (2017) Bushehr Elderly Health (BEH) programme: study protocol and design of musculoskeletal system and cognitive function (stage II). BMJ Open 7(8):e013606

18. Ostovar A, Nabipour I, Larijani B, Heshmat R, Darabi H, Vahdat $\mathrm{K}$ et al (2015) Bushehr Elderly Health (BEH) Programme, phase I (cardiovascular system). BMJ Open 5(12):e009597

19. Aadahl M, Andreasen AH, Hammer-Helmich L, Buhelt L, Jorgensen T, Glumer C (2013) Recent temporal trends in sleep duration, domain-specific sedentary behaviour and physical activity. A survey among 25-79-year-old Danish adults. Scand J Public Health 41(7):706-711

20. Aadahl M, Jorgensen T (2003) Validation of a new self-report instrument for measuring physical activity. Med Sci Sports Exerc 35(7):1196-1202

21. World Health Organization. WHO Criteria for Diagnosis of Osteoporosis. https://www.4bonehealth.org/education/world-healt h-organization-criteria-diagnosis-osteoporosis/. Accessed 25 Jan 2019

22. Cruz-Jentoft AJ, Baeyens JP, Bauer JM, Boirie Y, Cederholm T, Landi F et al (2010) Sarcopenia: European consensus on definition and diagnosis: Report of the European Working Group on Sarcopenia in Older People. Age Ageing 39(4):412-423

23. Chen LK, Liu LK, Woo J, Assantachai P, Auyeung TW, Bahyah KS et al (2014) Sarcopenia in Asia: consensus report of the Asian Working Group for Sarcopenia. J Am Med Dir Assoc 15(2):95-101

24. Shafiee G, Ostovar A, Heshmat R, Keshtkar AA, Sharifi F, Shadman $\mathrm{Z}$ et al (2018) Appendicular skeletal muscle mass reference values and the peak muscle mass to identify sarcopenia among Iranian healthy population. Int J Prev Med 9:25

25. American Heart Association, National Heart, Lung, and Blood Institute, Grundy SM, Cleeman JI, Daniels SR et al (2005) Diagnosis and management of the metabolic syndrome. An American Heart Association/National Heart, Lung, and Blood Institute Scientific Statement. Executive summary. Cardiol Rev 13(6):322-327

26. Hill KD, Farrier K, Russell M, Burton E (2017) Dysmobility syndrome: current perspectives. Clin Interv Aging 12:145-152

27. Carol S (2017) Intake: energy. In: Krause's food and the nutrition care process, 14th edn. Elsevier, Inc., New York

28. Kaji H (2014) Interaction between muscle and bone. J Bone Metab 21(1):29-40

29. Kang SY, Lim GE, Kim YK, Kim HW, Lee K, Park TJ et al (2017) Association between sarcopenic obesity and metabolic syndrome in postmenopausal women: a cross-sectional study based on the
Korean National Health and Nutritional Examination Surveys from 2008 to 2011. J Bone Metab 24(1):9-14

30. Yoo JI, Kim H, Ha YC, Kwon HB, Koo KH (2018) Osteosarcopenia in patients with hip fracture is related with high mortality. J Korean Med Sci 33(4):e27

31. Drey M, Sieber CC, Bertsch T, Bauer JM, Schmidmaier R, FiAT Intervention Group (2016) Osteosarcopenia is more than sarcopenia and osteopenia alone. Aging Clin Exp Res 28(5):895-899

32. Huo YR, Suriyaarachchi P, Gomez F, Curcio CL, Boersma D, Muir SW et al (2015) Phenotype of osteosarcopenia in older individuals with a history of falling. J Am Med Dir Assoc 16(4):290-295

33. Wang YJ, Wang Y, Zhan JK, Tang ZY, He JY, Tan P et al (2015) Sarco-osteoporosis: prevalence and association with frailty in Chinese community-dwelling older adults. Int J Endocrinol 2015:482940

34. Janssen I, Heymsfield SB, Allison DB, Kotler DP, Ross R (2002) Body mass index and waist circumference independently contribute to the prediction of nonabdominal, abdominal subcutaneous, and visceral fat. Am J Clin Nutr 75(4):683-688

35. Hirschfeld HP, Kinsella R, Duque G (2017) Osteosarcopenia: where bone, muscle, and fat collide. Osteoporos Int 28(10):2781-2790

36. Demontiero O, Boersma D, Suriyaarachchi P, Duque G (2014) Clinical outcomes of impaired muscle and bone interactions. Clin Rev Bone Miner Metab 12(2):86-92

37. Gusmao-Sena MH, Curvello-Silva K, Barreto-Medeiros JM, DaCunha-Daltro CH (2016) Association between sarcopenic obesity and cardiovascular risk: where are we? Nutr Hosp 33(5):592

38. Atkins JL, Whincup PH, Morris RW, Lennon LT, Papacosta O, Wannamethee SG (2014) Sarcopenic obesity and risk of cardiovascular disease and mortality: a population-based cohort study of older men. J Am Geriatr Soc 62(2):253-260

39. Inzerillo AM, Epstein S (2004) Osteoporosis and diabetes mellitus. Rev Endocr Metab Disord 5(3):261-268

40. Ho-Pham LT, Chau PMN, Do AT, Nguyen HC, Nguyen TV (2018) Type 2 diabetes is associated with higher trabecular bone density but lower cortical bone density: the Vietnam Osteoporosis Study. Osteoporos Int 29(9):2059-2067

41. Russell-Jones D, Khan R (2007) Insulin-associated weight gain in diabetes - causes, effects and coping strategies. Diabetes Obes Metab 9(6):799-812

42. Morley JE (2008) Diabetes, sarcopenia, and frailty. Clin Geriatr Med 24(3):455-469, vi

43. Plotnikoff RC, Taylor LM, Wilson PM, Courneya KS, Sigal RJ, Birkett N, Raine KI, Svenson LW (2006) Factors associated with physical activity in Canadian adults with diabetes. Med Sci Sports Exerc 38(8):1526-1534

44. De Vries H, van't Riet J, Spigt M, Metsemakers J, van den Akker M, Vermunt JK, Kremers S (2008) Clusters of lifestyle behaviors: results from the Dutch SMILE study. Prev Med 46(3):203-208

45. Al Saedi A, Hassan EB, Duque G (2019) The diagnostic role of fat in osteosarcopenia. J Lab Precis Med. https://doi.org/10.21037 /jlpm.2019.02.01

Publisher's Note Springer Nature remains neutral with regard to jurisdictional claims in published maps and institutional affiliations. 